\title{
Non-destructive evaluation of elastic properties, and ultrasonic attenuation of transverse wave of anisotropic and heterogeneous media
}

\author{
P.A. Bodian ${ }^{\text {a }}$, P. Guy ${ }^{\text {a }}$, B. Chassignole ${ }^{\text {b }}$, O.Dupont ${ }^{\text {b }}$ \\ a MATEIS CNRS UMR 5510, 7 avenue J. Capelle, F-69621Villeurbanne \\ b EDF R\&D Matériaux et Mécanique des Composants Site des Renardières pape- \\ arago.bodian@insa-lyon.fr
}

In industry to limit the number of experimental models in RD projects, to better understand and well interpret the complex ultrasonic phenomena observed during controls on site, simulations of ultrasonic controls are carried out. These simulations are more realistic than the description of structures to control is accurate, especially in terms of elastic constants, and intrinsic attenuation.

This study is dedicated to the adaptation of existing measurement techniques to coarse grains materiel characterization such as steels cast by centrifugation and alloys made of Nickel. The first aim of this survey is to improve knowledge on the influence of the metallurgical characteristics of anisotropic and heterogeneous materials on the ultrasonic propagation and the second is to enhance computing codes performances to trace reliably the elastic constants and ultrasonic attenuation from experimental measurements.

For this purpose, an experimental system for measuring on a single specimen, first the stiffness constants and second the attenuation in oblique incidence has been developed. The originality of this device is that it allows us to work beyond the longitudinal critical angle and therefore to measure the attenuation properties of transverse waves. To get elastic constants from the measured ultrasonic velocities, we will show the potential of global optimization algorithms such as genetic algorithms, less likely to converge on local minima of the function that we seek to minimize. Measurements of transverse waves attenuation by decomposition of the beam spectrum of plane waves are presented. 